\title{
Notes on the Global Well-Posedness for the Maxwell-Navier-Stokes System
}

\author{
Ensil Kang ${ }^{1}$ and Jihoon Lee ${ }^{2}$ \\ ${ }^{1}$ Department of Mathematics, Chosun University, Gwangju 501-759, Republic of Korea \\ ${ }^{2}$ Department of Mathematics, Chung-Ang University, Seoul 156-756, Republic of Korea
}

Correspondence should be addressed to Jihoon Lee; jhleepde@cau.ac.kr

Received 28 March 2013; Accepted 26 June 2013

Academic Editor: Changxing Miao

Copyright (c) 2013 E. Kang and J. Lee. This is an open access article distributed under the Creative Commons Attribution License, which permits unrestricted use, distribution, and reproduction in any medium, provided the original work is properly cited.

Masmoudi (2010) obtained global well-posedness for 2D Maxwell-Navier-Stokes system. In this paper, we reprove global existence of regular solutions to the 2D system by using energy estimates and Brezis-Gallouet inequality. Also we obtain a blow-up criterion for solutions to 3D Maxwell-Navier-Stokes system.

\section{Introduction}

In this paper, we consider Maxwell-Navier-Stokes equations in $\mathbb{R}^{d}(d=2,3)$ as follows:

$$
\begin{gathered}
\frac{\partial v}{\partial t}+(v \cdot \nabla) v-\Delta v+\nabla p=j \times B \quad \text { in } \mathbb{R}^{d} \times(0, T), \\
\frac{\partial E}{\partial t}-\nabla \times B=-j \quad \text { in } \mathbb{R}^{d} \times(0, T), \\
\frac{\partial B}{\partial t}+\nabla \times E=0 \quad \text { in } \mathbb{R}^{d} \times(0, T), \\
\nabla \cdot v=\nabla \cdot B=0 \quad \text { in } \mathbb{R}^{d} \times(0, T), \\
j=E+v \times B,
\end{gathered}
$$

subject to the initial data

$$
\begin{gathered}
v(x, 0)=v_{0}(x), \quad E(x, 0)=E_{0}(x), \\
B(x, 0)=B_{0}(x) .
\end{gathered}
$$

Here $v, E$, and $B: \mathbb{R}^{d} \times(0, T) \rightarrow \mathbb{R}^{3}$ are vector fields defined on $\mathbb{R}^{d}(d=2$ or 3$)$. Vector fields $v, E$, and $B$ denote fluid velocity, electric fields and magnetic fields, respectively. $p$ denotes the scalar pressure and $j$ is the electric current given by Ohm's law. $j \times B$ represents the Lorentz force. Here we put the viscosity and the electric resistivity to be 1 for the simplification. Note that in $2 \mathrm{D}$ case, vector fields $v, E$, and $B$ can be understood as $v(x, t)=\left(v_{1}\left(x_{1}, x_{2}, t\right), v_{2}\left(x_{1}, x_{2}, t\right), 0\right)$, and so forth.
For the compatibility of the initial data, we assume that

$$
\nabla \cdot v_{0}=\nabla \cdot B_{0}=0 .
$$

Since the divergence-free condition of the magnetic field is conserved, $\nabla \cdot B=0$ in (1) is not necessary in general if we assume the divergence-free condition for the initial data of the magnetic field in $\mathbb{R}^{d}$. In many physical situations, current displacement term $\partial_{t} E$ is neglected because the physical coefficient for this term is very small $\left(\sim 1 / c^{2}\right.$, where $c$ denotes the speed of light). But mathematically, the presence of the term $\partial_{t} E$ in the second equation (Ampere-Maxwell equation) preserves the hyperbolic nature of the Maxwell equation in the Maxwell-Navier-Stokes equations (see $[1,2]$ and references therein). Also we remark that full Maxwell-Navier-Stokes equations have been used for the accurate computation of electromagnetic hypersonics in aerothermodynamics (see [3, $4]$ and references therein). For further physical motivations, see [5].

Neglecting the current displacement term, MaxwellNavier-Stokes system is reduced to the usual MHD system. There have been many extensive mathematical studies for the existence, blow-up criterion, and regularity criterion of MHD and related models (see [6-12] and references therein). Recently, Maxwell-Navier-Stokes system has been receiving much mathematical attention after pioneering work of Masmoudi [2]. In [2], global existence of regular solutions to (1) in $\mathbb{R}^{2}$ is proved by using the Besov-type $\widetilde{L}$ space technique 
developed by Chemin and Lerner [13]. In [1, 14], the local existence of mild solution and the global existence of (1) with small data have been studied. Duan [15] studied large time behaviour of solutions to (1). In [16], Ibrahim and Yoneda obtained local-in-time existence for nondecaying initial data in torus. Also Germain and Masmoudi [17] studied global existence of solutions to Euler-Maxwell equations with small data and Jang and Masmoudi [18] mathematically derived Ohm's law from the kinetic equation.

The aim of this paper is to study the global well-posedness for (1) using the standard energy estimates. We obtain the local-in-time existence of $H^{2}$ solution by using the standard mollifier technique (see Proposition 4) and re-prove the global existence of $\mathrm{H}^{2}$ solution for 2D Maxwell-Navier-Stokes system (see Theorem 1) by using standard energy estimates and Brezis-Gallouet inequality, which was used to prove global existence of regular solution for the partial viscous Boussinesq equations by Chae [19]. Also we provide blow-up criterion of regular solutions to 3D Maxwell-Navier-Stokes equations (see Theorem 2).

We state our main results in the following.

Theorem 1. Assume that $\left(v_{0}, E_{0}, B_{0}\right) \in H^{2}\left(\mathbb{R}^{2}\right)$ and $\nabla \cdot v_{0}=$ $\nabla \cdot B_{0}=0$. Then, for any $T>0$, there exists a solution to 2D Maxwell-Navier-Stokes system (1) such that $(v, E, B) \in$ $C\left((0, T] ; H^{2}\right)$ and $(\nabla v, j) \in L^{2}\left(0, T ; H^{2}\right)$.

Theorem 2. Suppose that $\left(v_{0}, E_{0}, B_{0}\right) \in H^{2}\left(\mathbb{R}^{3}\right)$ and $\nabla \cdot v_{0}=\nabla$. $B_{0}=0$. If $T^{*}$, the maximal existence time of the local existence of regular solution to 3D Maxwell-Navier-Stokes system (1), is finite, then

$$
\int_{0}^{T^{*}}\|v(t)\|_{L^{\infty}}^{2}+\|B(t)\|_{L^{\infty}}^{8 / 3} d t=\infty .
$$

Remark 3. (1) As logarithmic inequality has been used in [2], Brezis-Gallouet inequality gives logarithmic-type estimates. But it provides double exponential bound compared with exponential bound in [2].

(2) The presence of the current displacement term $\partial_{t} E$ makes Maxwell-Navier-Stokes system do not enjoy the scaling invariance property of the usual Navier-Stokes system, $v_{\lambda}(x, t)=\lambda v\left(\lambda x, \lambda^{2} t\right)$. In Theorem $2, \int_{0}^{T}\|v(t)\|_{L^{\infty}}^{2} d t$ is concurrent with the usual scaling invariant norm of solutions to 3D Navier-Stokes equations.

The rest of this paper is organized as follows. In Section 2, we provide the local-in-time existence of regular solution to 2D and 3D Maxwell-Navier-Stokes systems and global existence of 2D Maxwell-Navier-Stokes system with large data. In Section 3, we provide the blow-up criterion for $\mathrm{H}^{2}$ solution to 3D Maxwell-Navier-Stokes system.

\section{Local Existence and Global Well-Posedness}

At first, we note that one can have the energy identity in two or three dimensions:

$$
\frac{1}{2} \frac{d}{d t}\left(\|v\|_{L^{2}}^{2}+\|B\|_{L^{2}}^{2}+\|E\|_{L^{2}}^{2}\right)+\|j\|_{L^{2}}^{2}+\|\nabla v\|_{L^{2}}^{2}=0 .
$$

The previously energy inequality can be justified for local in time regular solution in the following proposition. In the following, $C$ denotes a harmless constant which may change from one line to the other. We prove local-in-time existence of $\mathrm{H}^{2}$ solution using the standard energy estimates.

Proposition 4. Let $\left(u_{0}, E_{0}, B_{0}\right) \in H^{2}\left(\mathbb{R}^{d}\right)(d=2$ or 3$)$ with $\nabla \cdot u_{0}=\nabla \cdot B_{0}=0$. Then there exists $T=T\left(\left\|u_{0}\right\|_{H^{2}}\right.$, $\left.\left\|E_{0}\right\|_{H^{2}},\left\|B_{0}\right\|_{H^{2}}\right)$ such that there exists a unique solution $(u, E$, $B) \in L^{\infty}\left(0, T ; H^{2}\left(\mathbb{R}^{d}\right)\right) \cap \operatorname{Lip}\left(0, T ; L^{2}\right)$.

Proof. We use the mollifier method as described in [20]. Although the details are similar to [20], we provide some a priori estimates for the reader's sake. We consider the standard mollifier operator

$$
\mathscr{J}_{\epsilon} f=\rho_{\epsilon} * f, \quad \rho_{\epsilon}(\cdot)=\frac{1}{\epsilon^{d}} \rho\left(\frac{\cdot}{\epsilon^{d}}\right)
$$

where $\rho \in C_{0}^{\infty}\left(\mathbb{R}^{d}\right)$, and $\rho \geq 0, \int_{\mathbb{R}^{d}} \rho d x=1$.

We introduce the following regularized system of (1):

$$
\begin{array}{r}
\partial_{t} v^{\epsilon}+\mathscr{J}_{\epsilon}\left(\mathscr{J}_{\epsilon} v^{\epsilon} \cdot \nabla\right) \mathscr{J}_{\epsilon} v^{\epsilon}-\Delta \mathscr{J}_{\epsilon}^{2} v^{\epsilon}+\nabla p^{\epsilon} \\
=\mathscr{J}_{\epsilon}\left(\mathscr{J}_{\epsilon}^{2} j^{\epsilon} \times \mathscr{J}_{\epsilon} B^{\epsilon}\right) \quad \text { in } \mathbb{R}^{d} \times(0, T), \\
\partial_{t} E^{\epsilon}-\nabla \times \mathscr{J}_{\epsilon}^{2} B^{\epsilon}=-\mathscr{J}_{\epsilon}^{2} j^{\epsilon} \quad \text { in } \mathbb{R}^{d} \times(0, T), \\
\partial_{t} B^{\epsilon}+\nabla \times \mathscr{J}_{\epsilon}^{2} E^{\epsilon}=0 \quad \text { in } \mathbb{R}^{d} \times(0, T), \\
\nabla \cdot v^{\epsilon}=\nabla \cdot B^{\epsilon}=0 \quad \text { in } \mathbb{R}^{d} \times(0, T), \\
j^{\epsilon}=E^{\epsilon}+\mathscr{J}_{\epsilon} v^{\epsilon} \times \mathscr{J}_{\epsilon} B^{\epsilon},
\end{array}
$$

with initial data $\left(v_{0}^{\epsilon}, E_{0}^{\epsilon}, B_{0}^{\epsilon}\right)=\left(\mathscr{J}_{\epsilon} v_{0}, \mathscr{J}_{\epsilon} E_{0}, \mathscr{J}_{\epsilon} B_{0}\right)$.

Taking the $L^{2}$ inner product of $(7)_{1},(7)_{2}$, and $(7)_{3}$ with $v^{\epsilon}$, $E^{\epsilon}, B^{\epsilon}$, respectively, we obtain

$$
\begin{aligned}
\frac{1}{2} \frac{d}{d t}( & \left.\left\|v^{\epsilon}\right\|_{L^{2}}^{2}+\left\|E^{\epsilon}\right\|_{L^{2}}^{2}+\left\|B^{\epsilon}\right\|_{L^{2}}^{2}\right) \\
& +\left\|\nabla \mathscr{J}_{\epsilon} v^{\epsilon}\right\|_{L^{2}}^{2}+\left\|\mathscr{J}_{\epsilon} j^{\epsilon}\right\|_{L^{2}}^{2} \\
= & -\frac{1}{2} \int_{\mathbb{R}^{d}}\left(\mathscr{J}_{\epsilon} v^{\epsilon}\right) \cdot \nabla\left(\mathscr{J}_{\epsilon} v^{\epsilon}\right)^{2} d x \\
& +\int_{\mathbb{R}^{d}}\left(\nabla \times \mathscr{J}_{\epsilon} B^{\epsilon}\right) \cdot \mathscr{J}_{\epsilon} E^{\epsilon} d x \\
& -\int_{\mathbb{R}^{d}}\left(\nabla \times \mathscr{J}_{\epsilon} E^{\epsilon}\right) \cdot \mathscr{J}_{\epsilon} B^{\epsilon} d x \\
& +\int_{\mathbb{R}^{d}}\left(\mathscr{J}_{\epsilon}^{2} j^{\epsilon} \times \mathscr{J}_{\epsilon} B^{\epsilon}\right) \cdot \mathscr{J}_{\epsilon} v^{\epsilon} d x \\
& +\int_{\mathbb{R}^{d}} \mathscr{J}_{\epsilon}^{2} j^{\epsilon} \cdot\left(\mathscr{J}_{\epsilon} v^{\epsilon} \times \mathscr{J}_{\epsilon} B^{\epsilon}\right) d x=0 .
\end{aligned}
$$


We compute the derivative $D^{\alpha}, \alpha$ is a multi-index such that $|\alpha| \leq 2$, of (7), multiply them by $D^{\alpha} v^{\epsilon}, D^{\alpha} E^{\epsilon}$, and $D^{\alpha} B^{\epsilon}$, respectively, and integrate them over $\mathbb{R}^{d}$ to obtain

$$
\begin{aligned}
\frac{1}{2} \frac{d}{d t}( & \left.\left\|v^{\epsilon}\right\|_{H^{2}}^{2}+\left\|E^{\epsilon}\right\|_{H^{2}}^{2}+\left\|B^{\epsilon}\right\|_{H^{2}}^{2}\right) \\
& +\left\|\nabla \mathscr{J}_{\epsilon} v^{\epsilon}\right\|_{H^{2}}^{2}+\left\|\mathscr{J}_{\epsilon} j^{\epsilon}\right\|_{H^{2}}^{2} \\
\leq & C\left\|\mathscr{J}_{\epsilon} v^{\epsilon} \otimes \mathscr{J}_{\epsilon} v^{\epsilon}\right\|_{H^{2}}\left\|\nabla \mathscr{J}_{\epsilon} v^{\epsilon}\right\|_{H^{2}} \\
& +C\left\|\mathscr{J}_{\epsilon}^{2} j^{\epsilon} \times \mathscr{J}_{\epsilon} B^{\epsilon}\right\|_{H^{2}}\left\|\mathscr{J}_{\epsilon} v^{\epsilon}\right\|_{H^{2}} \\
& +C\left\|\mathscr{F}_{\epsilon}^{2} j^{\epsilon}\right\|_{H^{2}}\left\|\mathscr{J}_{\epsilon} v^{\epsilon} \times \mathscr{J}_{\epsilon} B^{\epsilon}\right\|_{H^{2}} \\
\leq & C\left(\left\|\mathscr{J}_{\epsilon} v^{\epsilon}\right\|_{H^{2}}^{4}+\left\|\mathscr{J}_{\epsilon} B^{\epsilon}\right\|_{H^{2}}^{4}\right) \\
& +\frac{1}{2}\left(\left\|\mathscr{J}_{\epsilon} \nabla v^{\epsilon}\right\|_{H^{2}}^{2}+\left\|\mathscr{J}_{\epsilon} j^{\epsilon}\right\|_{H^{2}}^{2}\right) .
\end{aligned}
$$

In the previously mentioned, $\mathscr{J}_{\epsilon} v^{\epsilon} \otimes \mathscr{J}_{\epsilon} v^{\epsilon}$ denotes a tensor $\left(\mathscr{J}_{\epsilon} v_{i}^{\epsilon} \mathscr{J}_{\epsilon} v_{j}^{\epsilon}\right)_{1 \leq j \leq d}$.

Using Picard's theorem, these estimates imply local existence of solution.

The main ingredient of the proof of Theorem 1 is the following Brezis-Gallouet inequality (logarithmic Sobolev inequality):

$$
\begin{array}{r}
\|f\|_{L^{\infty}} \leq C\left(1+\|f\|_{L^{2}}+\|\nabla f\|_{L^{2}}\left(\log ^{+}\|\Delta f\|_{L^{2}}\right)^{1 / 2}\right), \\
f \in H^{2}\left(\mathbb{R}^{2}\right) .
\end{array}
$$

Here $\log ^{+} a$ denotes $\log (e+a)$.

Proof of Theorem 1. We provide a priori estimates on the regular solutions. Let $T$ be a finite maximal time of existence in Proposition 4. By obtaining $H^{2}$ bound on $(0, T]$ of solution, we can continue solution beyond $T$ by using Proposition 4 .

Taking curl operator on $(1)_{1}$ and $\partial_{i}=\partial / \partial x_{i}(i=1,2)$ operator on $(1)_{2}$ and $(1)_{3}$, we have

$$
\begin{gathered}
\frac{\partial \omega}{\partial t}+(v \cdot \nabla) \omega-\Delta \omega=\nabla \times(j \times B), \quad \text { in } \mathbb{R}^{2} \times(0, T), \\
\frac{\partial\left(\partial_{i} E\right)}{\partial t}-\nabla \times \partial_{i} B=-\partial_{i} j, \quad \text { in } \mathbb{R}^{2} \times(0, T) \\
\frac{\partial\left(\partial_{i} B\right)}{\partial t}+\nabla \times \partial_{i} E=0, \quad \text { in } \mathbb{R}^{2} \times(0, T)
\end{gathered}
$$

(i) $H^{1}$ Estimates. Taking scalar product (11) with $\omega, \partial_{i} E$, and $\partial_{i} B$, respectively, and summing over $i=1,2$, we have

$$
\begin{gathered}
\frac{1}{2} \frac{d}{d t}\|\omega\|_{L^{2}}^{2}+\|\nabla \omega\|_{L^{2}}^{2}=\int_{\mathbb{R}^{2}} \nabla \times(j \times B) \cdot \omega d x, \\
\frac{1}{2} \frac{d}{d t}\|\nabla E\|_{L^{2}}^{2}=\sum_{i} \int_{\mathbb{R}^{2}} \nabla \times \partial_{i} B \cdot \partial_{i} E d x-\int_{\mathbb{R}^{2}} \nabla j \cdot \nabla E d x \\
\frac{1}{2} \frac{d}{d t}\|\nabla B\|_{L^{2}}^{2}=-\sum_{i} \int_{\mathbb{R}^{2}} \nabla \times \partial_{i} E \cdot \partial_{i} B d x .
\end{gathered}
$$

Using the identity

$$
\int_{\mathbb{R}^{2}} \nabla \times \partial_{i} B \cdot \partial_{i} E d x=\int_{\mathbb{R}^{2}} \nabla \times \partial_{i} E \cdot \partial_{i} B d x
$$

and $E=j-v \times B$, we obtain

$$
\begin{gathered}
\frac{1}{2} \frac{d}{d t}\left(\|\nabla E\|_{L^{2}}^{2}+\|\nabla B\|_{L^{2}}^{2}\right)+\|\nabla j\|_{L^{2}}^{2} \\
=\int_{\mathbb{R}^{2}} \nabla j \cdot \nabla(v \times B) d x .
\end{gathered}
$$

In the following, $\epsilon$ denotes a sufficiently small positive number. Since it holds that $\nabla \times(j \times B)=(B \cdot \nabla) j$, we estimate the right-hand side of (12) using Young's inequality and interpolation inequality:

$$
\begin{aligned}
& \left|\int_{\mathbb{R}^{2}} \nabla \times(j \times B) \cdot \omega d x\right| \\
& \quad \leq\|B\|_{L^{4}}\|\nabla j\|_{L^{2}}\|\omega\|_{L^{4}} \\
& \quad \leq C\|B\|_{L^{2}}^{1 / 2}\|\nabla B\|_{L^{2}}^{1 / 2}\|\omega\|_{L^{2}}^{1 / 2}\|\nabla \omega\|_{L^{2}}^{1 / 2}\|\nabla j\|_{L^{2}} \\
& \quad \leq C\|\omega\|_{L^{2}}^{2}\|\nabla B\|_{L^{2}}^{2}+\epsilon\|\nabla \omega\|_{L^{2}}^{2}+\epsilon\|\nabla j\|_{L^{2}}^{2},
\end{aligned}
$$

where $\epsilon$ is a small positive number. Also we have

$$
\begin{aligned}
& \left|\int_{\mathbb{R}^{2}} \nabla j \cdot \nabla(v \times B) d x\right| \\
& \leq \int_{\mathbb{R}^{2}}|\nabla j||v||\nabla B| d x \\
& \quad+\int_{\mathbb{R}^{2}}|\nabla j||B||\nabla v| d x=I+I I .
\end{aligned}
$$

We estimate

$$
\begin{gathered}
I \leq C\|v\|_{L^{\infty}}^{2}\|\nabla B\|_{L^{2}}^{2}+\epsilon\|\nabla j\|_{L^{2}}^{2} \\
I I \leq\|B\|_{L^{4}}\|\nabla v\|_{L^{4}}\|\nabla j\|_{L^{2}} \leq C\|B\|_{L^{2}}^{2}\|\nabla v\|_{L^{2}}^{2}\|\nabla B\|_{L^{2}}^{2} \\
+\epsilon\|\Delta v\|_{L^{2}}^{2}+\epsilon\|\nabla j\|_{L^{2}}^{2} .
\end{gathered}
$$

Collecting previous estimates, we have

$$
\begin{aligned}
& \frac{d}{d t}\left(\|\omega\|_{L^{2}}^{2}+\|\nabla E\|_{L^{2}}^{2}+\|\nabla B\|_{L^{2}}^{2}\right) \\
& \quad+\|\nabla \omega\|_{L^{2}}^{2}+\|\nabla j\|_{L^{2}}^{2} \leq C\|\omega\|_{L^{2}}^{2}\|\nabla B\|_{L^{2}}^{2} \\
& \quad+C\|v\|_{L^{\infty}}^{2}\|\nabla B\|_{L^{2}}^{2}+C\|\nabla B\|_{L^{2}}^{2}\|\nabla v\|_{L^{2}}^{2}\|\nabla B\|_{L^{2}}^{2} .
\end{aligned}
$$


(ii) $H^{2}$ Estimates. Taking $\Delta$ operator on $(1)_{1},(1)_{2}$, and $(1)_{3}$ and $L^{2}$ scalar product with $\Delta v, \Delta E$, and $\Delta B$, respectively, we have

$$
\begin{aligned}
& \frac{1}{2} \frac{d}{d t}\|\Delta v\|_{L^{2}}^{2}+\|\nabla \Delta v\|_{L^{2}}^{2} \\
& \leq C \int_{\mathbb{R}^{2}}|\nabla v|\left|D^{2} v\right| d x \\
& \quad+\int_{\mathbb{R}^{2}}|\Delta(j \times B)||\Delta v| d x:=I_{1}+I_{2}, \\
& \frac{1}{2} \frac{d}{d t}\left(\|\Delta E\|_{L^{2}}^{2}+\|\Delta B\|_{L^{2}}^{2}\right)+\|\Delta j\|_{L^{2}}^{2} \\
& \leq \int_{\mathbb{R}^{2}}|\Delta j||\Delta(v \times B)| d x:=I_{3} .
\end{aligned}
$$

We estimate $I_{1}, I_{2}$, and $I_{3}$ using interpolation inequality, Young's inequality, and Hölder's inequality:

$$
\begin{aligned}
I_{1} \leq C\|\nabla v\|_{L^{4}}\|\Delta v\|_{L^{4}}\|\Delta v\|_{L^{2}} \\
\leq C\|\nabla v\|_{L^{2}}^{1 / 2}\|\Delta v\|_{L^{2}}^{3 / 2}\|\nabla \Delta v\|_{L^{2}}^{1 / 2} \\
\leq C\|\nabla v\|_{L^{2}}^{2 / 3}\|\Delta v\|_{L^{2}}^{2}+\epsilon\|\nabla \Delta v\|_{L^{2}}^{2}, \\
I_{2} \leq C \int_{\mathbb{R}^{2}}|\nabla j||\nabla B||\Delta v| d x \\
\quad+C \int_{\mathbb{R}^{2}}|\Delta j||B||\Delta v| d x \\
+C \int_{\mathbb{R}^{2}}|j||\Delta B||\Delta v| d x:=I_{21}+I_{22}+I_{23} .
\end{aligned}
$$

Each term can be estimated by the standard interpolation inequality and Young's inequality as follows:

$$
\begin{aligned}
I_{21} & \leq C\|\nabla j\|_{L^{4}}\|\nabla B\|_{L^{4}}\|\Delta v\|_{L^{2}} \\
& \leq C\|j\|_{L^{2}}^{1 / 4}\|\Delta j\|_{L^{2}}^{3 / 4}\|B\|_{L^{2}}^{1 / 4}\|\Delta B\|_{L^{2}}^{3 / 4}\|\nabla v\|_{L^{2}}^{1 / 2}\|\nabla \Delta v\|_{L^{2}}^{1 / 2} \\
& \leq C\|j\|_{L^{2}}^{2 / 3}\|B\|_{L^{2}}^{2 / 3}\|\nabla v\|_{L^{2}}^{4 / 3}\|\Delta B\|_{L^{2}}^{2}+\epsilon\|\Delta j\|_{L^{2}}^{2} \\
& \leq C\left(\|j\|_{L^{2}}^{2}+\|\nabla v\|_{L^{2}}^{2}\right)\|\Delta B\|_{L^{2}}^{2}+\epsilon\|\Delta j\|_{L^{2}}^{2}, \\
I_{22} & \leq C\|\Delta j\|_{L^{2}}\|B\|_{L^{2}}\|\Delta v\|_{L^{4}} \\
& \leq C\|\Delta j\|_{L^{2}}\|B\|_{L^{2}}^{3 / 4}\|\Delta B\|_{L^{2}}^{1 / 4}\|\Delta v\|_{L^{2}}^{1 / 2}\|\nabla \Delta v\|_{L^{2}}^{1 / 2} \\
& \leq \epsilon\|\Delta j\|_{L^{2}}^{2}+C\|\Delta B\|_{L^{2}}^{1 / 2}\|\Delta v\|_{L^{2}}^{1 / 2}\|\nabla \Delta v\|_{L^{2}} \\
& \leq \epsilon\|\Delta j\|_{L^{2}}^{2}+\epsilon\|\nabla \Delta v\|_{L^{2}}^{2}+C\|j\|_{L^{2}}\|\nabla v\|_{L^{2}}\|\Delta B\|_{L^{2}}^{2}, \\
I_{23} & \leq C\|j\|_{L^{\infty}}\|\Delta B\|_{L^{2}}\|\Delta v\|_{L^{2}} \\
& \leq C\|j\|_{L^{2}}\|\Delta j\|_{L^{2}}^{1 / 2}\|\Delta B\|_{L^{2}}\|\nabla v\|_{L^{2}}^{1 / 2}\|\nabla \Delta v\|_{L^{2}}^{1 / 2} \\
& \leq \epsilon\|\Delta j\|_{L^{2}}^{2}+C\|j\|_{L^{2}}^{2 / 3}\|\Delta B\|_{L^{2}}^{4 / 3}\|\nabla v\|_{L^{2}}^{2 / 3}\|\nabla \Delta v\|_{L^{2}}^{2 / 3} \\
& \leq \epsilon\|\Delta j\|_{L^{2}}^{2}+\epsilon\|\nabla \Delta v\|_{L^{2}}^{2}+C\|j\|_{L^{2}}\|\nabla v\|_{L^{2}}\|\Delta B\|_{L^{2}}^{2} .
\end{aligned}
$$

$I_{3}$ can be written as

$$
\begin{aligned}
I_{3} \leq & C \int_{\mathbb{R}^{2}}|\Delta j||\nabla v||\nabla B| d x \\
& +C \int_{\mathbb{R}^{2}}|\Delta j||\Delta v||B| d x \\
& +C \int_{\mathbb{R}^{2}}|\Delta j||v||\Delta B| d x:=I_{31}+I_{32}+I_{33}, \\
I_{31} \leq & C\|\Delta j\|_{L^{2}}\|\nabla v\|_{L^{4}}\|\nabla B\|_{L^{4}} \\
\leq & C\|\Delta j\|_{L^{2}}\|\nabla v\|_{L^{2}}^{3 / 4}\|\nabla \Delta v\|_{L^{2}}^{1 / 4}\|B\|_{L^{2}}^{1 / 4}\|\Delta B\|_{L^{2}}^{3 / 4} \\
\leq & \epsilon \Delta j\left\|_{L^{2}}^{2}+\epsilon\right\| \nabla \Delta v\left\|_{L^{2}}^{2}+C\right\| \nabla v\left\|_{L^{2}}^{2}\right\| \Delta B \|_{L^{2}}^{2 .}
\end{aligned}
$$

The same as the estimate of $I_{22}$, we obtain

$$
\begin{aligned}
I_{32} \leq & \epsilon\|\Delta j\|_{L^{2}}^{2}+\epsilon\|\nabla \Delta v\|_{L^{2}}^{2} \\
& +C\|j\|_{L^{2}}\|\nabla v\|_{L^{2}}\|\Delta B\|_{L^{2}}^{2} .
\end{aligned}
$$

Also we have

$$
I_{33} \leq C\|\Delta j\|_{L^{2}}\|v\|_{L^{\infty}}\|\Delta B\|_{L^{2}} \leq \epsilon\|\Delta j\|_{L^{2}}^{2}+C\|v\|_{L^{\infty}}^{2}\|\Delta B\|_{L^{2}}^{2} .
$$

Therefore, we have

$$
\begin{aligned}
\frac{d}{d t}( & \left.\|\Delta v\|_{L^{2}}^{2}+\|\Delta E\|_{L^{2}}^{2}+\|\Delta B\|_{L^{2}}^{2}\right) \\
& +\|\nabla \Delta v\|_{L^{2}}^{2}+\|\Delta j\|_{L^{2}}^{2} \\
\leq & C\left(1+\|\nabla v\|_{L^{2}}^{2}+\|j\|_{L^{2}}^{2}+\|v\|_{L^{\infty}}^{2}\right) \\
& \times\left(\|\Delta v\|_{L^{2}}^{2}+\|\Delta B\|_{L^{2}}^{2}\right) .
\end{aligned}
$$

(iii) Use of Brezis-Gallouet Inequality. Using Brezis-Gallouet inequality, we obtain

$$
\begin{aligned}
\frac{d}{d t}( & \left.\|\Delta v\|_{L^{2}}^{2}+\|\Delta E\|_{L^{2}}^{2}+\|\Delta B\|_{L^{2}}^{2}\right) \\
& +\|\nabla \Delta v\|_{L^{2}}^{2}+\|\Delta j\|_{L^{2}}^{2} \\
\leq & C\left(1+\|\nabla v\|_{L^{2}}^{2}+\|j\|_{L^{2}}^{2}+\|v\|_{L^{2}}^{2}+\|\nabla v\|_{L^{2}}^{2}\right) \\
& \times\left(\|\Delta v\|_{L^{2}}^{2}+\|\Delta E\|_{L^{2}}^{2}+\|\Delta B\|_{L^{2}}^{2}\right) \\
& \times \log ^{+}\left(\|\Delta v\|_{L^{2}}^{2}+\|\Delta E\|_{L^{2}}^{2}+\|\Delta B\|_{L^{2}}^{2}\right) .
\end{aligned}
$$

Let $y(t)=\|\Delta v\|_{L^{2}}^{2}+\|\Delta E\|_{L^{2}}^{2}+\|\Delta B\|_{L^{2}}^{2}$, and let $z(t)=1+$ $\|\Delta v\|_{L^{2}}^{2}+\|j\|_{L^{2}}^{2}+\|v\|_{L^{2}}^{2}+\|\nabla v\|_{L^{2}}^{2}$. Hence one has

$$
\frac{d}{d t} y(t) \leq C z(t) y(t) \log ^{+} y(t) \text {. }
$$

Since

$$
\int_{0}^{T} z(t) d t \leq C(1+T),
$$


the bound of $y(t)$ is immediate as follows:

$$
\begin{aligned}
\sup _{0 \leq t \leq T}( & \left.\|\Delta v\|_{L^{2}}^{2}+\|\Delta E\|_{L^{2}}^{2}+\|\Delta B\|_{L^{2}}^{2}\right) \\
\leq & \left(\left\|\Delta v_{0}\right\|_{L^{2}}^{2}+\left\|\Delta E_{0}\right\|_{L^{2}}^{2}+\left\|\Delta B_{0}\right\|_{L^{2}}^{2}\right) \\
& \times \exp (\exp (C(T+1))) .
\end{aligned}
$$

This completes the proof of Theorem 1.

\section{Blow-Up Criterion for 3D Maxwell-Navier-Stokes System}

In this section, we provide a blow-up criterion for $H^{2}$ solution in Proposition 4 to 3D Maxwell-Navier-Stokes system.

Proof of Theorem 2. Assume that

$$
\int_{0}^{T^{*}}\|v(t)\|_{L^{\infty}}^{2}+\|B(t)\|_{L^{\infty}}^{8 / 3} d t<\infty
$$

where $T^{*}$ is the finite maximal existence time of a classical solution.

Similar to the computation in Section 2, one has $H^{1}$ estimates of $E$ and $B$ as follows:

$$
\begin{aligned}
\frac{1}{2} \frac{d}{d t} & \left(\|\nabla E\|_{L^{2}}^{2}+\|\nabla B\|_{L^{2}}^{2}\right)+\|\nabla j\|_{L^{2}}^{2} \\
& =\int_{\mathbb{R}^{3}} \nabla j \cdot \nabla(v \times B) d x \\
& \leq C\|\nabla(v \times B)\|_{L^{2}}^{2}+\epsilon\|\nabla j\|_{L^{2}}^{2} \\
& \leq C\|B\|_{L^{\infty}}^{2}\|\nabla v\|_{L^{2}}^{2}+C\|v\|_{L^{\infty}}^{2}\|\nabla B\|_{L^{2}}^{2}+\epsilon\|\nabla j\|_{L^{2}}^{2} .
\end{aligned}
$$

$H^{1}$ estimates of $v$ are as follows:

$$
\begin{aligned}
\frac{1}{2} \frac{d}{d t}\|\nabla v\|_{L^{2}}^{2}+\|\Delta v\|_{L^{2}}^{2} \\
\leq \int_{\mathbb{R}^{3}}|v||\nabla v||\Delta v| d x \\
\quad+\int_{\mathbb{R}^{3}}|j \times B||\Delta v| d x \\
\leq C\|v\|_{L^{\infty}}^{2}\|\nabla v\|_{L^{2}}^{2} \\
\quad+C\|j \times B\|_{L^{2}}^{2}+\epsilon\|\Delta v\|_{L^{2}}^{2} .
\end{aligned}
$$

The estimate of $\|j \times B\|_{L^{2}}^{2}$ is provided in the following:

$$
\begin{aligned}
\|j \times B\|_{L^{2}}^{2} & \leq C\|E \times B\|_{L^{2}}^{2}+C\|(v \times B) \times B\|_{L^{2}}^{2} \\
& \leq C\|E\|_{L^{2}}^{2}\|B\|_{L^{\infty}}^{2}+C\|v\|_{L^{6}}^{2}\|B\|_{L^{6}}^{4} \\
& \leq C\|E\|_{L^{2}}^{2}\|B\|_{L^{\infty}}^{2}+C\|\nabla v\|_{L^{2}}^{2}\|B\|_{L^{2}}^{4 / 3}\|B\|_{L^{\infty}}^{8 / 3} .
\end{aligned}
$$

Thus we have

$$
\begin{aligned}
& \frac{d}{d t}(\left.\|\nabla v\|_{L^{2}}^{2}+\|\nabla E\|_{L^{2}}^{2}+\|\nabla B\|_{L^{2}}^{2}\right) \\
&+\|\Delta v\|_{L^{2}}^{2}+\|\nabla j\|_{L^{2}}^{2} \\
& \leq C\left(1+\|v\|_{L^{\infty}}^{2}+\|B\|_{L^{\infty}}^{8 / 3}\right) \\
& \quad \times\left(\|\nabla v\|_{L^{2}}^{2}+\|\nabla E\|_{L^{2}}^{2}+\|\nabla B\|_{L^{2}}^{2}\right)+C\|B\|_{L^{\infty}}^{2} .
\end{aligned}
$$

Gronwall's inequality gives us that

$$
\|(\nabla v, \nabla E, \nabla B)\|_{L^{\infty}\left(0, T^{*} ; L^{2}\right)}^{2}+\|(\Delta v, \nabla j)\|_{L^{2}\left(0, T^{*} ; L^{2}\right)}^{2} \leq C<\infty .
$$

Next, we consider $H^{2}$ estimates.

Integrating by parts and using Young's inequality, it follows that

$$
\begin{aligned}
& \frac{1}{2} \frac{d}{d t}\left(\|\Delta E\|_{L^{2}}^{2}+\|\Delta B\|_{L^{2}}^{2}\right) \\
&+\|\Delta j\|_{L^{2}}^{2} \leq C\|\Delta(v \times B)\|_{L^{2}}^{2}+\epsilon\|\Delta j\|_{L^{2}}^{2} \\
& \leq C\|\Delta v\|_{L^{2}}^{2}\|B\|_{L^{\infty}}^{2}+C\|v\|_{L^{\infty}}^{2}\|\Delta B\|_{L^{2}}^{2} \\
&+C\|\nabla v\|_{L^{4}}^{2}\|\nabla B\|_{L^{4}}^{2}+\epsilon\|\Delta j\|_{L^{2}}^{2} \\
& \leq C\|\Delta v\|_{L^{2}}^{2}\|B\|_{L^{\infty}}^{2}+C\|v\|_{L^{\infty}}^{2}\|\Delta B\|_{L^{2}}^{2} \\
&+C\|v\|_{L^{\infty}}\|B\|_{L^{\infty}}\|\Delta v\|_{L^{2}}\|\Delta B\|_{L^{2}}+\epsilon\|\Delta j\|_{L^{2}}^{2} .
\end{aligned}
$$

Similarly, it follows that

$$
\begin{aligned}
\frac{1}{2} \frac{d}{d t} \| & \Delta v\left\|_{L^{2}}^{2} \leq C\right\| \nabla(v \cdot \nabla v) \|_{L^{2}}^{2} \\
& +C\|\nabla(j \times B)\|_{L^{2}}^{2}+\epsilon\|\nabla \Delta v\|_{L^{2}}^{2} \\
\leq & C\|\nabla v\|_{L^{4}}^{4}+C\|v\|_{L^{\infty}}^{2}\|\Delta v\|_{L^{2}}^{2} \\
& +C\|\nabla E\|_{L^{6}}^{2}\|B\|_{L^{3}}^{2}+C\|E\|_{L^{6}}^{2}\|\nabla B\|_{L^{3}}^{2} \\
& +C\|\nabla(v \times B)\|_{L^{2}}^{2}\|B\|_{L^{\infty}}^{2}+C\|v \times B\|_{L^{3}}^{2}\|\nabla B\|_{L^{6}}^{2} .
\end{aligned}
$$

Using the interpolation inequality, one has

$$
\|\nabla v\|_{L^{4}}^{4} \leq C\|v\|_{L^{\infty}}^{2}\|\Delta v\|_{L^{2}}^{2} .
$$

Interpolation inequality and Young's inequality produce that

$$
\begin{aligned}
& \|E\|_{L^{6}}^{2}\|\nabla B\|_{L^{3}}^{2} \\
& \quad \leq C\|\nabla E\|_{L^{2}}^{2}\|\nabla B\|_{L^{2}}\|\Delta B\|_{L^{2}} \\
& \quad \leq C\|\nabla E\|_{L^{2}}^{2}\left(\|\nabla B\|_{L^{2}}^{2}+\|\Delta B\|_{L^{2}}^{2}\right) .
\end{aligned}
$$

Similarly, we estimate that

$$
\begin{aligned}
& \|\nabla(v \times B)\|_{L^{2}}^{2}\|B\|_{L^{\infty}}^{2} \\
& \quad \leq C\left(\|\nabla v\|_{L^{3}}^{2}\|\nabla B\|_{L^{2}}^{2}+\|\nabla v\|_{L^{2}}^{2}\|\nabla B\|_{L^{3}}^{2}\right), \\
& \|v \times B\|_{L^{3}}^{2}\|\nabla B\|_{L^{6}}^{2} \leq C\|v\|_{L^{\infty}}^{2}\|B\|_{L^{3}}^{2}\|\Delta B\|_{L^{2}}^{2} .
\end{aligned}
$$


We already know that

$$
\|(\nabla v, \nabla E, \nabla B)\|_{L^{\infty}\left(0, T^{*} ; L^{2}\right)}^{2}<C .
$$

Gathering all the estimates, we achieve

$$
\begin{aligned}
\frac{d}{d t}( & \left.\|\Delta v\|_{L^{2}}^{2}+\|\Delta E\|_{L^{2}}^{2}+\|\Delta B\|_{L^{2}}^{2}\right) \\
& +\|\nabla \Delta v\|_{L^{2}}^{2}+\|\Delta j\|_{L^{2}}^{2} \\
\leq & C\left(1+\|v\|_{L^{\infty}}^{2}+\|B\|_{L^{\infty}}^{2}\right) \\
& \times\left(1+\|\Delta v\|_{L^{2}}^{2}+\|\Delta E\|_{L^{2}}^{2}+\|\Delta B\|_{L^{2}}^{2}\right) .
\end{aligned}
$$

Using Gronwall's inequality, we conclude that

$$
\|(\Delta v, \Delta E, \Delta B)\|_{L^{\infty}\left(0, T^{*} ; L^{2}\right)}^{2}+\|(\nabla \Delta v, \Delta j)\|_{L^{2}\left(0, T^{*} ; L^{2}\right)}^{2} \leq C<\infty .
$$

This completes the proof of Theorem 2 .

\section{Acknowledgments}

Ensil Kang's work was supported by research fund from Chosun University, 2009, and Jihoom Lee's work was partly supported by the National Research Foundation of Korea (NRF-2011-0006697) and Chung-Ang University Research Grants in 2013.

\section{References}

[1] P. Germain, S. Ibrahim, and N. Masmoudi, "On the wellposedness of the Navier-Stokes-Maxwell system," http://arxiv .org/abs/1207.6187.

[2] N. Masmoudi, "Global well posedness for the Maxwell-NavierStokes system in 2D," Journal de Mathématiques Pures et Appliquées. Neuvième Série, vol. 93, no. 6, pp. 559-571, 2010.

[3] D. D’Ambrosio and D. Giordano, "Electromagnetic fluid dynamics for aerospace applications. Part I: classification and critical review of physical models," in Proceedings of the 35th AIAA Plasmadynamics and Lasers Conference, Portland, Ore, USA, June 2004, AIAA Paper 2004-2165.

[4] D. D’Ambrosio, M. Pandol, and D. Giordano, "Electromagnetic fluid dynamics for aerospace applications. Part II: numerical simulations using different physical models," in Proceedings of the 35th AIAA Plasmadynamics and Lasers Conference, Portland, Ore, USA, June 2004, AIAA Paper 2004-2362.

[5] P. A. Davidson, An Introduction to Magnetohydrodynamics, Cambridge Texts in Applied Mathematics, Cambridge University Press, Cambridge, UK, 2001.

[6] C. Cao and J. Wu, "Two regularity criteria for the 3D MHD equations," Journal of Differential Equations, vol. 248, no. 9, pp. 2263-2274, 2010.

[7] C. Cao and J. Wu, "Global regularity for the 2D MHD equations with mixed partial dissipation and magnetic diffusion," Advances in Mathematics, vol. 226, no. 2, pp. 1803-1822, 2011.

[8] G. Duvaut and J.-L. Lions, "Inéquations en thermoélasticité et magnétohydrodynamique," Archive for Rational Mechanics and Analysis, vol. 46, pp. 241-279, 1972.
[9] Z. Lei and Y. Zhou, "BKM's criterion and global weak solutions for magnetohydrodynamics with zero viscosity," Discrete and Continuous Dynamical Systems. Series A, vol. 25, no. 2, pp. 575583, 2009.

[10] M. Sermange and R. Temam, "Some mathematical questions related to the MHD equations," Communications on Pure and Applied Mathematics, vol. 36, no. 5, pp. 635-664, 1983.

[11] J. Wu, "Regularity criteria for the generalized MHD equations," Communications in Partial Differential Equations, vol. 33, no. 13, pp. 285-306, 2008.

[12] Y. Zhou, "Regularity criteria for the generalized viscous MHD equations," Annales de l'Institut Henri Poincaré. Analyse Non Linéaire, vol. 24, no. 3, pp. 491-505, 2007.

[13] J.-Y. Chemin and N. Lerner, "Flot de champs de vecteurs non lipschitziens et équations de Navier-Stokes," Journal of Differential Equations, vol. 121, no. 2, pp. 314-328, 1995.

[14] S. Ibrahim and S. Keraani, "Global small solutions for the Navier-Stokes-Maxwell system," SIAM Journal on Mathematical Analysis, vol. 43, no. 5, pp. 2275-2295, 2011.

[15] R. Duan, "Green's function and large time behavior of the Navier-Stokes-Maxwell system," Analysis and Applications, vol. 10, no. 2, pp. 133-197, 2012.

[16] S. Ibrahim and T. Yoneda, "Local solvability and loss of smoothness of the Navier-Stokes-Maxwell equations with large initial data," Journal of Mathematical Analysis and Applications, vol. 396, no. 2, pp. 555-561, 2012.

[17] P. Germain and N. Masmoudi, "Global existence for the EulerMaxwell system," http://arxiv.org/abs/1107.1595.

[18] J. Jang and N. Masmoudi, "Derivation of Ohm's law from the kinetic equations," SIAM Journal on Mathematical Analysis, vol. 44, no. 5, pp. 3649-3669, 2012.

[19] D. Chae, "Global regularity for the $2 \mathrm{D}$ Boussinesq equations with partial viscosity terms," Advances in Mathematics, vol. 203, no. 2, pp. 497-513, 2006.

[20] A. J. Majda and A. L. Bertozzi, Vorticity and Incompressible Flow, vol. 27 of Cambridge Texts in Applied Mathematics, Cambridge University Press, Cambridge, UK, 2002. 


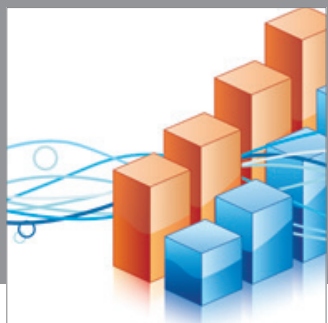

Advances in

Operations Research

mansans

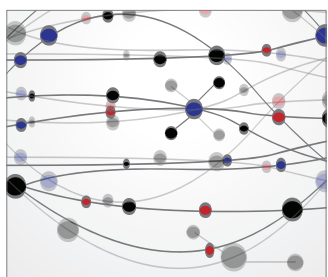

The Scientific World Journal
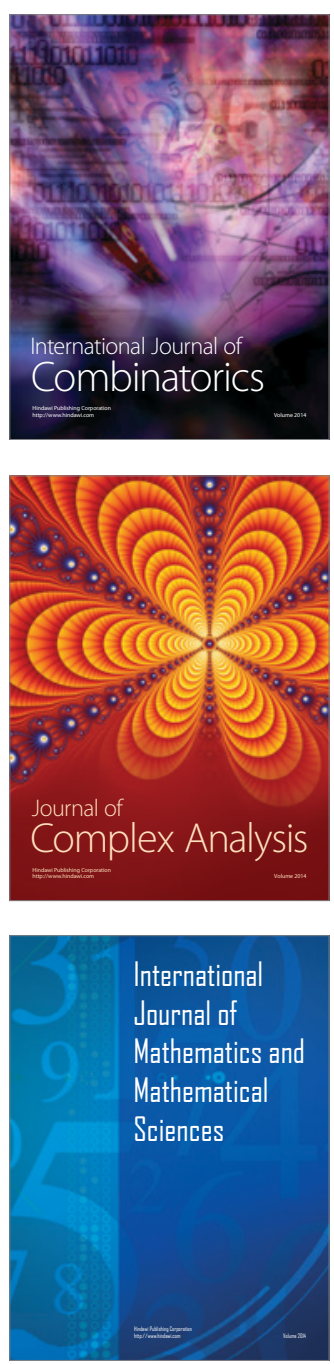
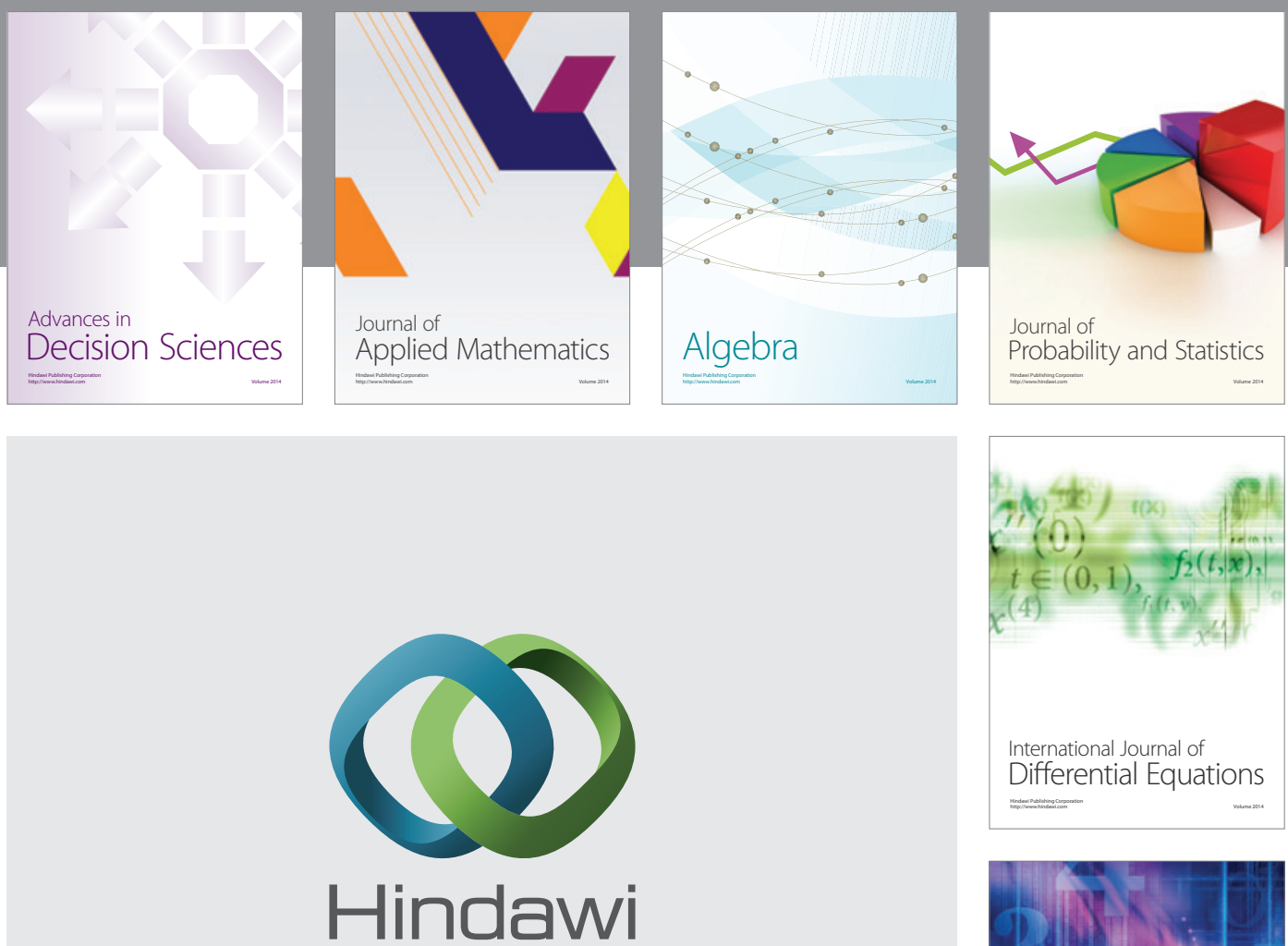

Submit your manuscripts at http://www.hindawi.com
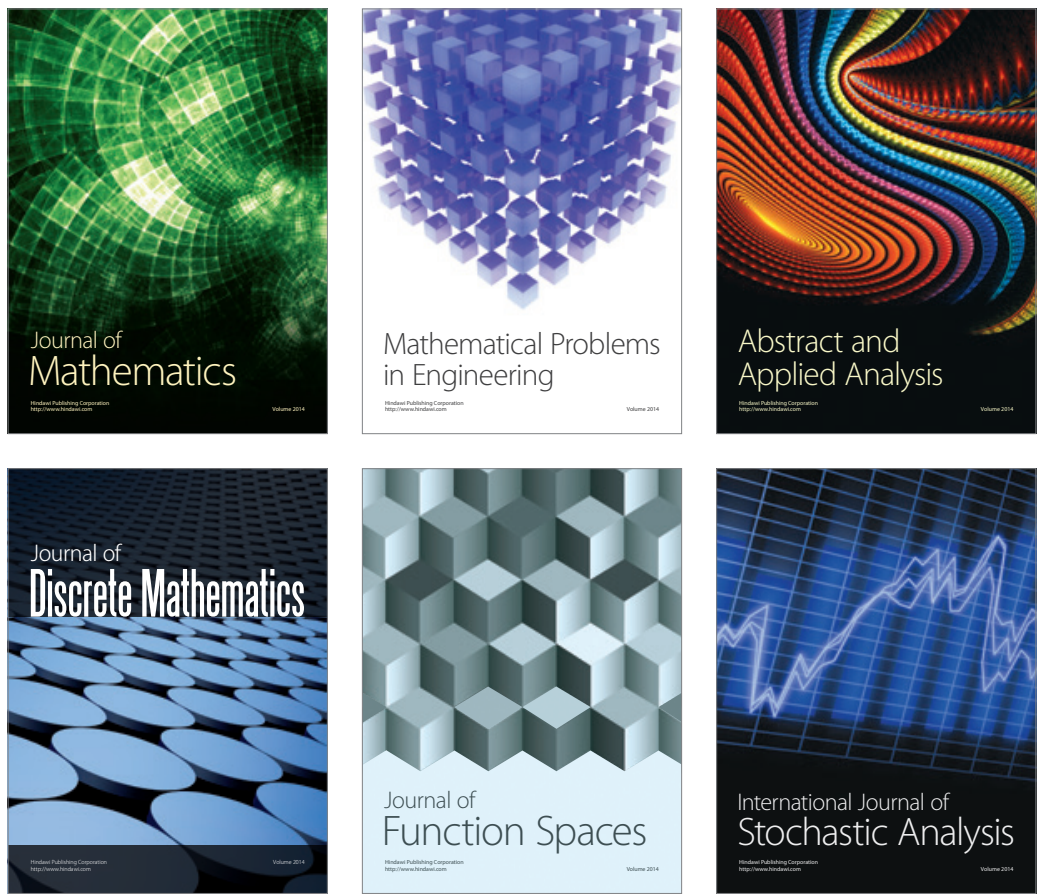

Journal of

Function Spaces

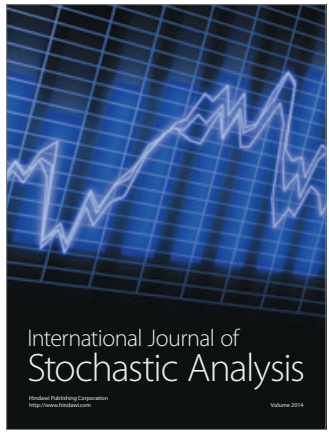

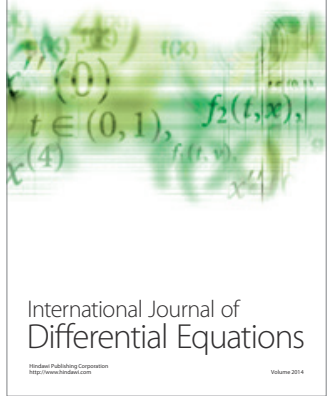
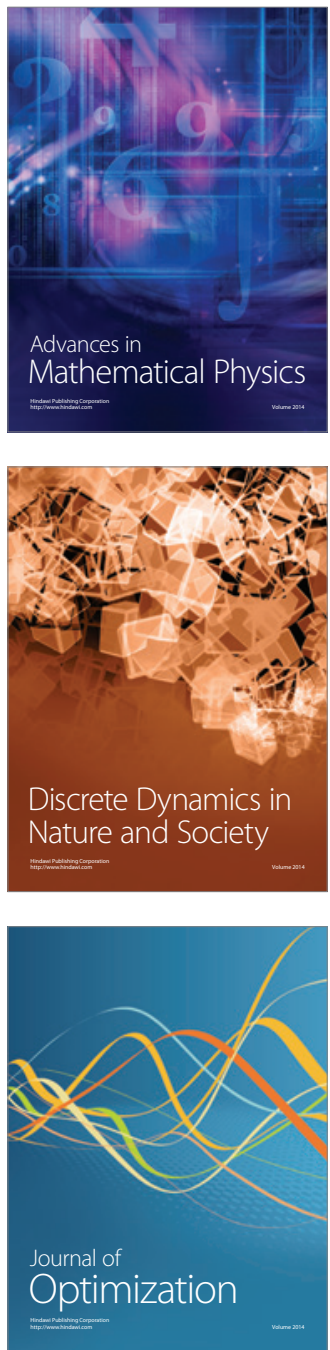